\title{
Image Optimization in a Computed-Radiography/Photostimulable-Phosphor System
}

\author{
Robert H. Sherrier, Harrell G. Chotas, G. Allan Johnson, Caroline Chiles, and Carl E. Ravin
}

\begin{abstract}
Photostimulable phosphor imaging is an exciting new technology that has several advantages over film/ screen radiography, the most important of which is the linearity of the photostimulable phosphor system over a wide exposure latitude. The photostimulable phosphor image is digital, and as such, provides options of how the image is viewed by radiologists. This report discusses the various image-processing parameters available for a photostimulable phosphor system and describes a rational approach for selecting these parameters in portable chest radiography. As photostimulable phosphor imaging becomes more widely implemented, an understanding of the processing parameters will facilitate the production of images that take full advantage of the benefits of these systems.
\end{abstract}

(C) 1989 by W.B. Saunders Company.

KEY WORDS: digital image processing, photostimulable phosphors.

$\mathbf{P}$ HOTOSTIMULABLE phosphor imaging is an exciting new technology that has the potential to improve image quality in diagnostic radiology. The primary advantage is that the image receptor, a photostimulable phosphor plate, unlike film/screen systems, is linear over a wide exposure latitude. ${ }^{1}$ The photostimulable phosphor technology results in a digital image that can be stored on magnetic or optical disks, transported within or between hospitals over high-speed transmission lines, or processed to enhance selected features. Photostimulable phosphor imaging has been used clinically since the early 1980s and is now available at a number of institutions. ${ }^{2-6}$ As more radiology departments acquire these units, an understanding of the technology becomes important. In particular, if these digital images are to be interpreted by radiologists on a film-based medium, the parameters used for image enhancement should not only be understood by the radiologists, but in many cases, selected by them.

From the Department of Radiology, Duke University Medical Center, Durham, NC.

Address reprint requests to Robert $H$. Sherrier, $M D, B o x$ 3808, Duke University Medical Center, Durham, NC 27710. () 1989 by W.B. Saunders Company. 0897-1889/89/0204-0003\$03.00/0
Unlike film/screen systems, the image receptor in photostimulable phosphor imaging is separate from the display medium. The photostimulable phosphor plate is exposed with conventional radiographic techniques, producing a latent image on the plate. This latent image is scanned by a small beam from a laser that stimulates the phosphor to emit visible light proportional to the exposure in the latent image. The stimulated emission is captured and digitized, yielding an image that can then be viewed on monitors or printed on film with a laser printer. The photostimulable phosphor images in this report were acquired with a Philips Computed Radiography (PCR)/photostimulable-phosphor system (Shelton, CT), and film was selected as the primary output medium for investigation.

The choice of film as the display medium has the advantage of familiarity to radiologists. Because of the linearity of the PCR plate receptor, the digital image can be adjusted so that consecutive films of patients have similar optical densities, regardless of variable exposure techniques. Disadvantages of a film-only system include the nonlinearity of the film itself and the inability to interactively adjust the contrast and brightness interactively or manipulate image-processing (edge-enhancement) parameters.

For a portable chest examination, exposure of the $14 \times 17$-in photostimulable-phosphor plate results in a single, unprocessed digital chest image that is stored on a magnetic or optical disc. With the PCR system, this image is printed in two different presentations on a single $10 \times$ 14-in film (these two images will subsequently be referred to as the left and right images). The left and right images are independently processed to achieve the desired gray-scale contrast and edge enhancement. While photostimulable phosphor systems have preselected (default) parameters that define the type and amount of image processing for a given examination, these parameters may not be optimal for all institutions and may therefore require modification.

The goal of this report is to describe a method for systematically selecting image-processing pa- 
rameters in a computed-radiography/photostimulable-phosphor system. The available parameters are described, and their impact on defined clinical tasks is discussed. While this report focuses on the PCR system and portable chest radiography, the principles can be applied to other digital radiography systems and other examinations.

\section{MATERIALS AND METHODS}

Portable chest radiographs were obtained with a PCR photostimulable phosphor system. A $14 \times 17$-inch PCR imaging plate was used for the portable chest images, which results in a $1,760 \times 2,140$-pixel digital image 10 bits deep. Analysis and manipulation of images in this report was simplified by the existence of a research network centered about SUN 3 (Sun Microsystems, Mountain View, CA) and Macintosh II (Apple Computer, Inc, Cupertino, CA) work stations. The PCR, a DuPont (Wilmington, DE) FD-2000 film digitizer, and all of the work stations are linked via an Ethernet network, permitting ready transfer of digital images between systems. Images are displayed on 19-in SUN grayscale monitors that display $1,152 \times 900$ lines, eight bits deep. Conventional portable radiographs at this institution are obtained with Kodak XL film in dual-screen cassettes using Kodak Lanex Regular (Rochester, NY) screens. A Humanoid Systems (Carson, CA) chest phantom and aluminum calibration step wedges were used for the measurements.

Imaging processing and enhancement of a digital image is accomplished by two basic image transforms: (1) manipulation of gray-scale and (2) edge enhancement. In the PCR there are four parameters for gray-scale adjustment and three parameters for edge enhancement. Both sets of parameters are used to convert the unprocessed ("raw") pixel values in the image to output pixel values that are then directly related to optical density on the film. The relationship between pixel value and optical density is linear over a range of 0.3 to 2.5 optical density units.

The image gray-scale transform parameters used in the PCR are graphically illustrated in Fig 1 and consist of the following: (1) GT-curve type; this may be linear $(\mathrm{GT}=A)$ or sigmoidal (GT $=B$ through $O$ ); (2) GC-center of rotation; this is the point on the curve about which the curve may be rotated; (3) GA-rotation amount (slope of the curve); the slope, in effect, determines the contrast in the image; and (4) GS - optical density offset shift of the curve; this allows the optical density of the entire image to be shifted.

The PCR system uses unsharp masking for edge enhancement. Several steps are involved in producing an unsharpmask image. First, a square area of adjacent pixels are averaged at each point in the image to obtain a blurred (low-frequency) image. The size of the square area (in pixels) about each pixel is called the kernel size, from convolution kernel, a structure used in many digital-smoothing techniques. Selection of the kernel size determines the size of the objects that are enhanced. A small kernel will differentially edge-enhance fine structures, whereas a large kernel will enhance larger structures. The blurred image is subtracted from the original to achieve a high-frequency image. The high-frequency image is then multiplied by a weighting factor (gain) and added back to the original image. The degree of enhancement can be adjusted as a function of the pixel value in the original image, as defined by a weighting factor curve, which will be described. In the PCR, the kernel size is specified indirectly using the frequency rank parameter (RN), the degree of enhancement (gain) with the RE parameter, and the weighting factor curve with the RT parameter.

The PCR defines several weighting factor curves for edge enhancement of the image. These curves are arbitrarily assigned the labels $F$ (flat) and $P$ through $V$. These curves control the amount of edge enhancement applied to different anatomical regions in the image. Figure 2 illustrates three
Fig 1. Types of gray-scale output transform curves and associated parameters. Curve type (PCR parameter: GT) may be linear or sigmoidal. The curves may be rotated about a selected center (GC) by a specified amount (GA), and then shifted a specified optical density offset (GS).

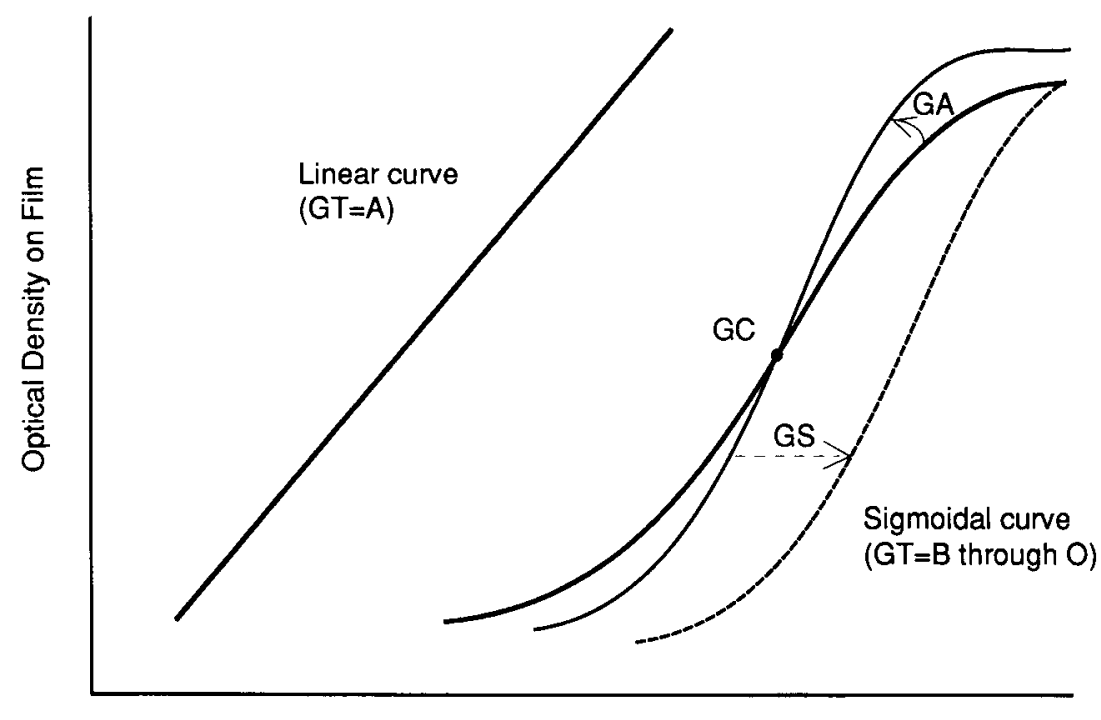

Pixel Value 


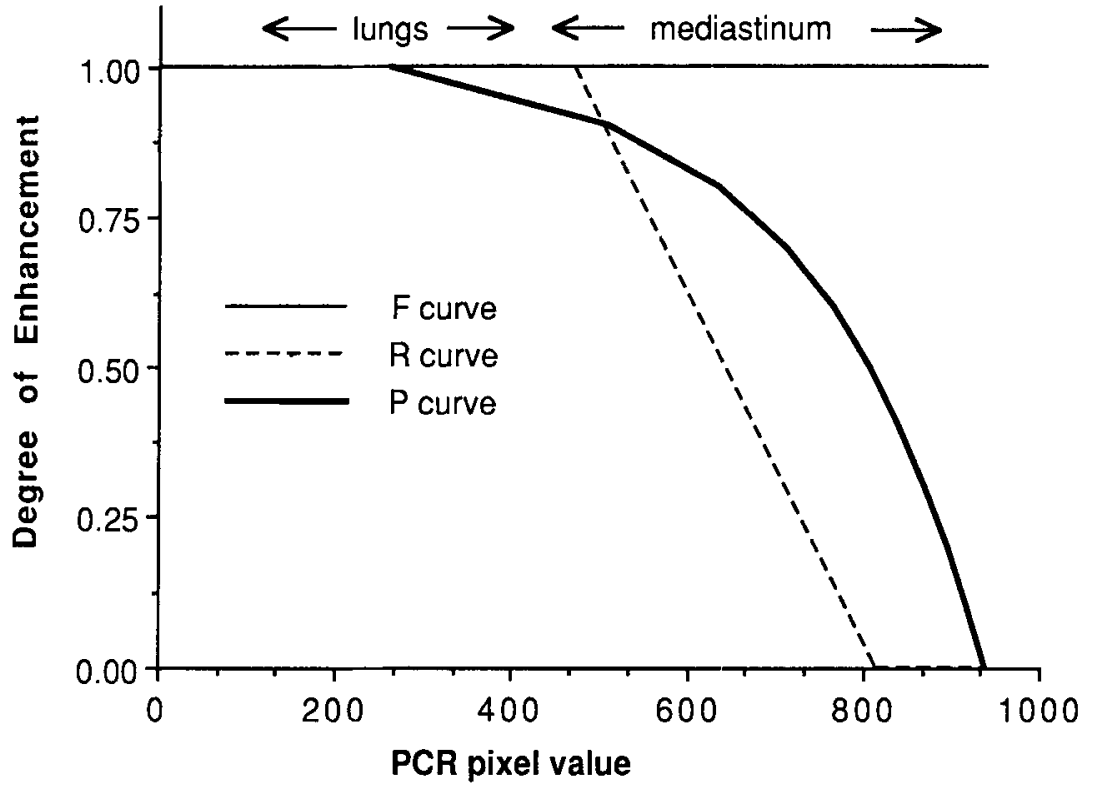

Fig 2. Weighting factor curves for the unsharp-masking algorithm (PCR parameter: RT). The flat PCR F-curve $(R T=F)$ provides full onhancement at all pixel values. The PCR default right image uses the R-curve (dashed line), which provides full enhancement for pixel values less than 475 (the lungs) and less enhancement in the mediastinum. The PCR P-curve provides more edge enhancement than the R-curve in the retrocardiac/subdiaphragmatic areas (pixel values $\mathbf{5 0 0}$ to 800 ) for increased visualization of catheters.
PCR weighting factor (RT) curves. A flat weighting factor curve ( $F$ curve) produces full edge enhancement at all pixels in the image. The R-curve also provides full edge enhancement at all pixel values less than 475 (in the lungs) but less enhancement in the retrocardiac and subdiaphragmatic areas. The pixel values for the heart/lung interface are approximately 500 to 600 , and those for an intracardiac catheter are typically 700 to 800 . The $P$ curve offers relatively more edge enhancement than the $R$ curve in the retrocardiac and subdiaphragmatic areas, with only slightly less enhancement in the lung region.

\section{Left and Right Images}

We have chosen to produce a left image that is similar to an ideally exposed conventional radiograph. This is the primary image that the radiologist interprets. It should have grayscale adjustments and edge-enhancement modifications that result in an image that is as close as possible to an ideally exposed conventional radiograph. The right image can be processed in such a manner that it provides information not available from the left image. For example, in portable chest radiography, edge-enhancement and gray-scale settings should enhance visualization of catheters and tubes as well as improve detection of pneumothoraces.

The first goal was to identify the PCR image-processing parameters that would produce a left image similar to a conventional portable chest radiograph. These parameters should be selected so that a similarly exposed PCR plate and conventional film/screen combination result in images having similar optical densities. An aluminum step wedge was initially used as an imaging target in this experiment; however, the PCR reader uses a variable gain, and some assumptions on the input dynamic range and the image histogram are used to set this gain. Because the aluminum step wedge did not reflect the physical characteristics of a chest, the results were inconsistent. There were additional concerns about different energy responses of the PCR plates and the conventional film/screen system after filtration by an aluminum step wedge. This led us to choose the humanoid chest phantom as the calibration standard.

Seven regions of interest on the films of the humanoid chest phantom were used as reference points for optical-density measurements. A conventional XL portable film and a PCR digital image of the humanoid chest phantom were obtained at $100 \mathrm{kV}$ (peak), $0.8 \mathrm{mAs}$, and 48 in SI distance. The unprocessed digital PCR image was transmitted to the SUN workstations, and the pixel values corresponding to the seven anatomical locations were measured using standard region-ofinterest utilities. The relationship between the optical density on the XL film versus the corresponding pixel value in the PCR image was then plotted. Inspection of this curve allowed selection of the best gray-scale curve type (PCR parameter: GT) and orientation (GC and GA) of the gray-scale output transform. The optical density offset (GS) was then chosen to align the optical densities of the conventional XL film and the PCR film.

The goal of the gray-scale and edge-enhancement adjustments for the right image is to increase visualization of support and/or monitoring tubes and catheters, especially in the mediastinum. Other sharp interfaces, such as a pneumothorax, should also be exaggerated to increase detection. One solution is to increase local contrast by choosing a gray-scale output transform with a steeper slope. While this will enhance the visibility of structures locally, similar to the selection of lung or soft-tissue windows in chest computed tomography images, it will also sacrifice contrast in other regions. Unsharp masking is a type of edge enhancement that increases local contrast without sacrificing dynamic range. Because increased visualization of edges is necessary in both the darker regions (for pneumothoraces and more peripheral catheters) and lighter regions (cardiac pacer wires, etc), edge enhancement should be performed in both of these regions.

In addition to the edge-enhancement options for the right image, a gray-scale output transform must also be selected. In the left image this curve is typically sigmoidal (simulating 
the characteristic curve of film when used as a detector), which provides less contrast for structures in the mediastinum than in the lungs. Because the goal of the right image is to increase contrast in both the lungs and mediastinum, a linear transform (PCR parameter $\mathrm{GT}=A$ ) with a wide latitude is advisable. While this type of curve by itself would provide less contrast in the lungs than in the left image, the edge enhancement of the unsharp-masking algorithm will

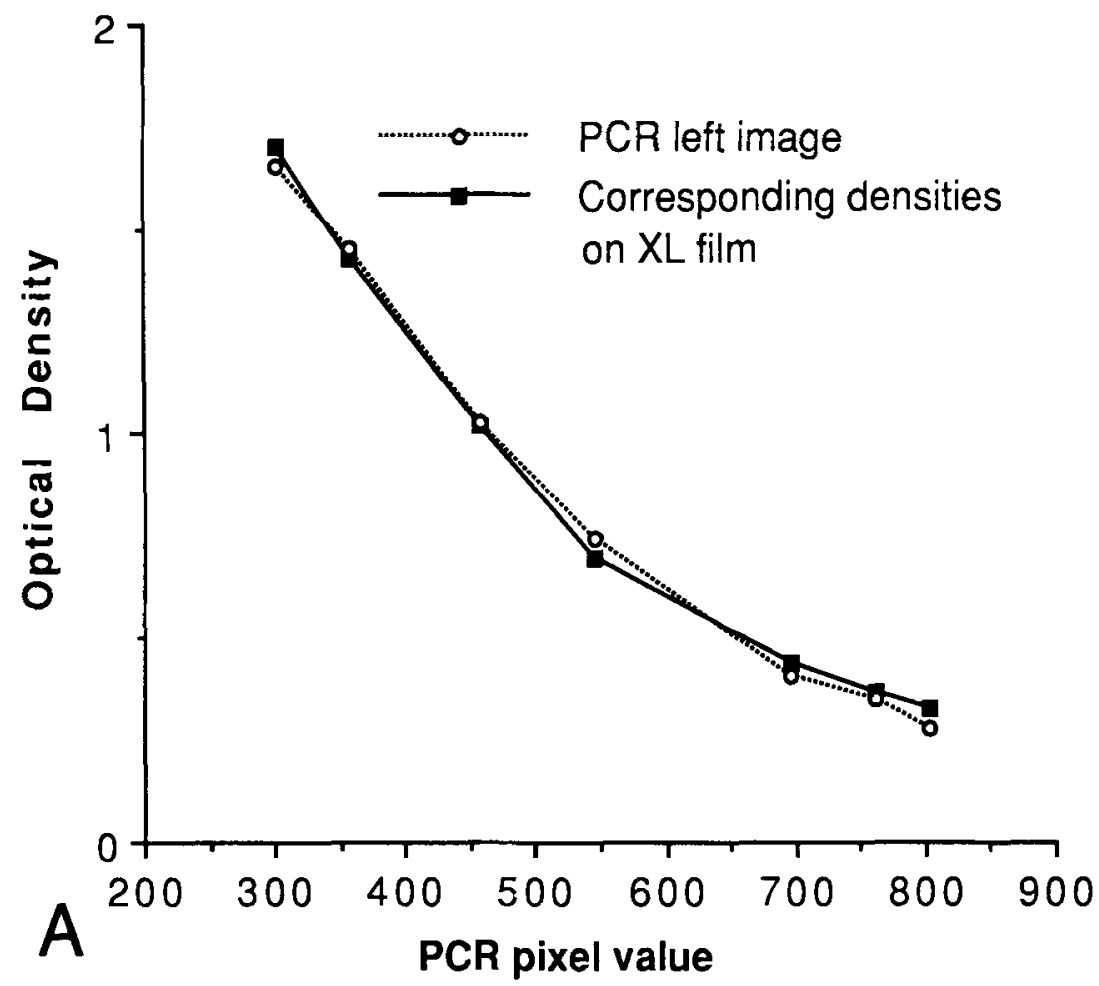

Fig 3. Gray-scale output transform curves for the left image. (A) Optical densities of the seven reference points on the humanoid chest phantom versus pixel value at the corresponding locations in the digital PCR image. The gray-scale transform that best matches the XL film is PCR curve type $G T=D$ (a sigmoidal curve). slope $G A=0.9$, center of rotation $\mathrm{GC}=1.5$, and optical density offset GS $=-0.15$. (B) Comparison of the optical densities on the chest phantom: the default PCR parameters versus our derived paremeters in Fig $3 A$.

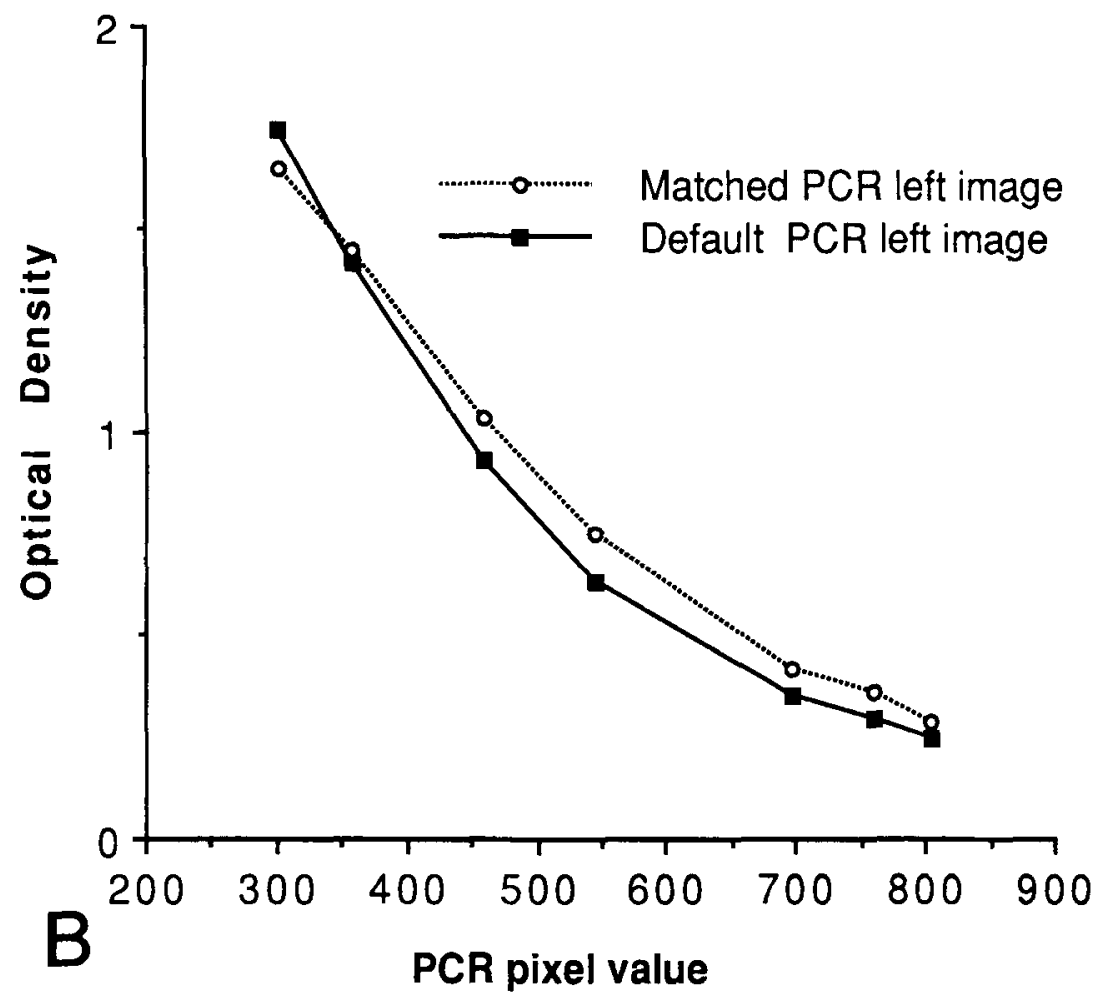


increase local contrast. The combination of a linear output transform and unsharp masking in both the lungs and mediastinum will provide increased contrast in both of these regions while preserving the dynamic range of the image.

\section{RESULTS}

Processing parameters for the left image are chosen to achieve optical densities (gray-scale contrast) that match an ideally exposed conventional radiograph. Figure $3 \mathrm{~A}$ illustrates the relationship between the PCR pixel values (as measured from reference points on the chest phantom) in the derived left image and the optical density measurements from the printed PCR film. In addition, the optical densities from the conventional radiograph (XL film) are plotted for each of the seven reference points. The PCR image was produced with a sigmoidal contrast curve type $(\mathrm{PCR}$ parameter $\mathrm{GT}=D)$, a slope $\mathrm{GA}=$
0.9 , rotation center $\mathrm{GC}=1.5$, and optical density offset GS $=-.15$, resulting in a match of optical densities for the reference points on the chest phantom ranging from 0.2 to 1.7 . This curve specification is significantly different from the PCR settings as delivered by the manufacturer $(\mathrm{GT}=E, \mathrm{GA}=1.0, \mathrm{GC}=1.6, \mathrm{GS}=$ -.20 ). Figure $3 \mathrm{~B}$ illustrates the differences in the optical densities in two PCR films, one produced using our derived (XL-matching) parameters and the other using the manufacturer's defaults. Figure 4 is the PCR left image, printed using the new parameters. The seven points used to make the measurements are indicated.

Edge enhancement (used in the right image) requires selection of the unsharp-masking kernel size or frequency rank (PCR parameter $R N$ ), weighting factor curve (RT), and degree of edge

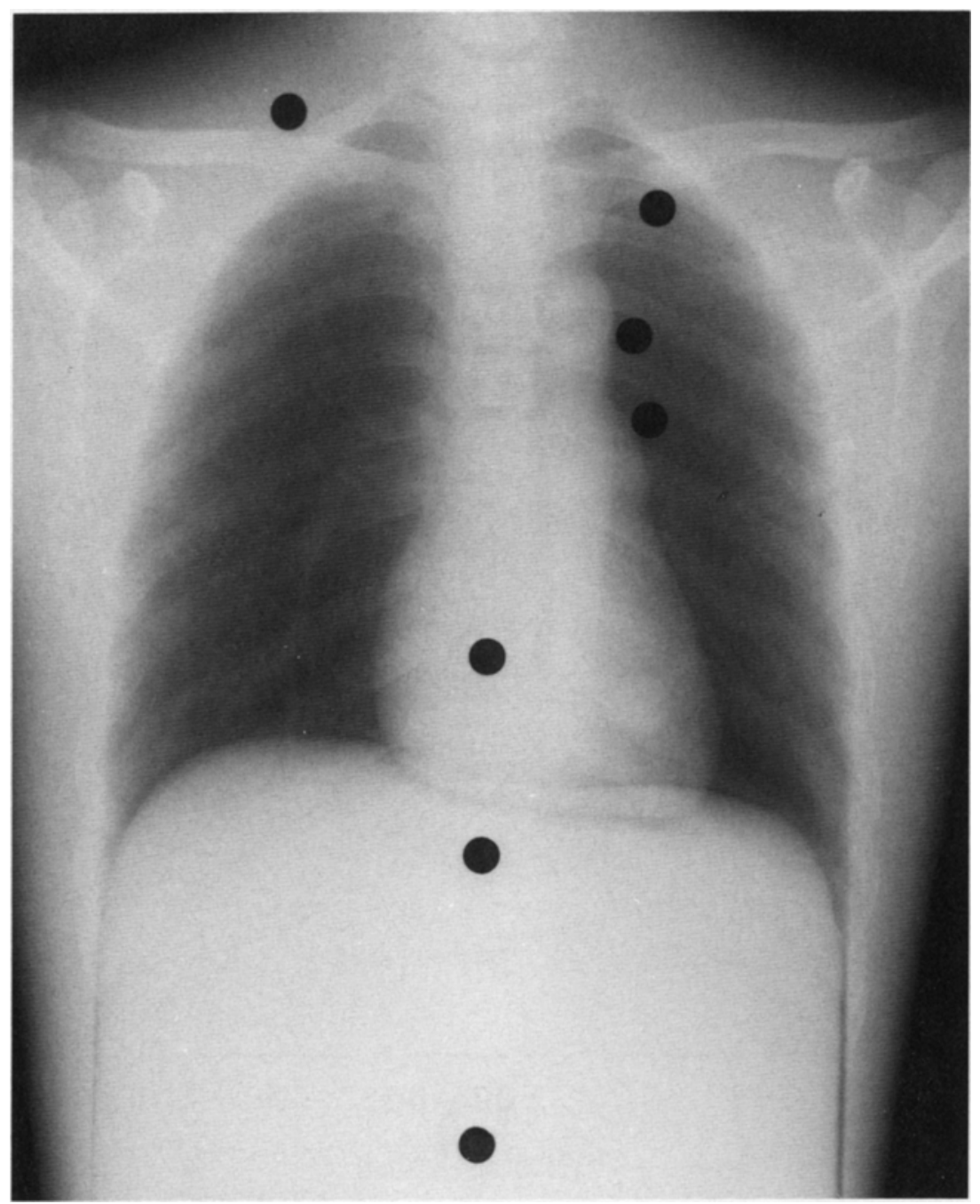

Fig 4. A PCR portable radiograph of the chest phantom after application of the derived parameters for the left image. Circles indicate the seven points used for the density measurements. 

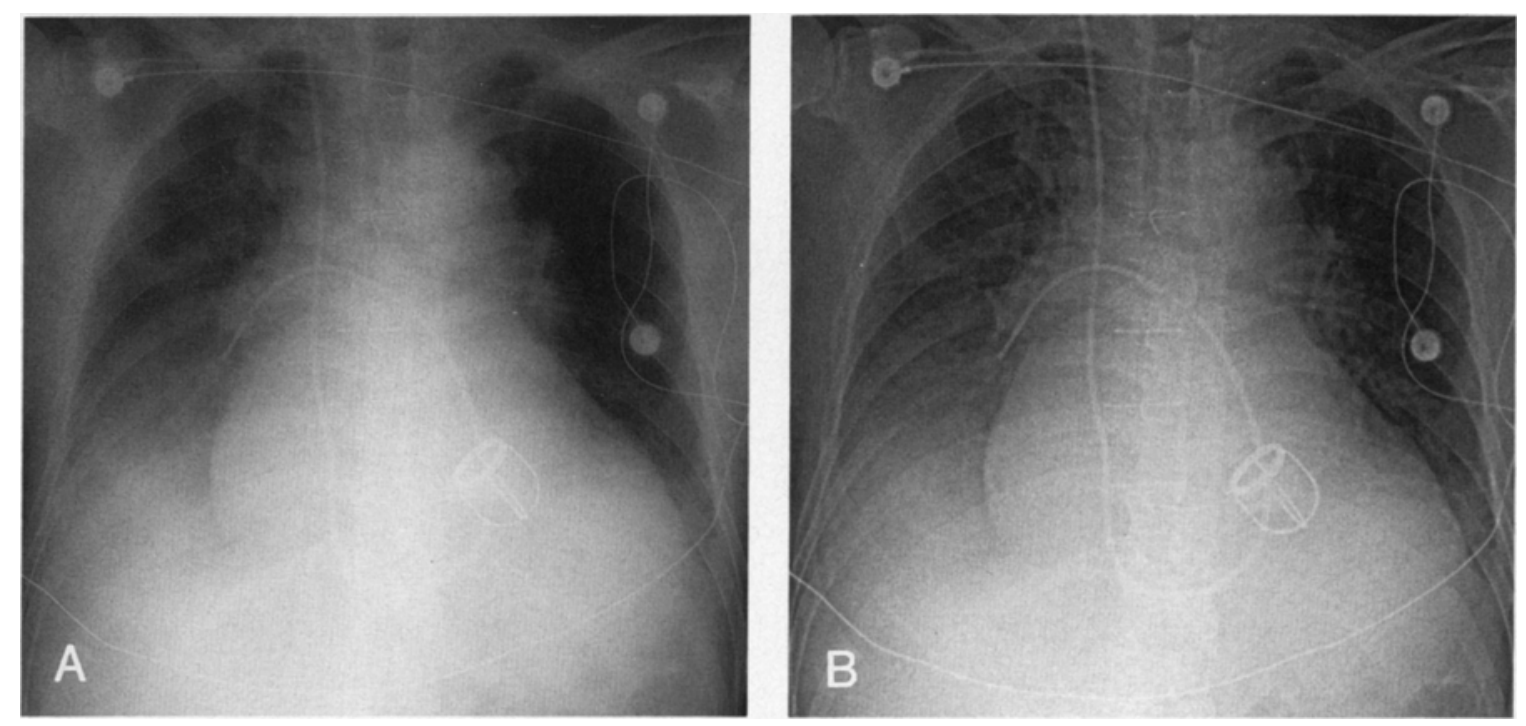

Fig 5. Unsharp masking. Effect of kernel size (frequency rank). (A) Kernel size $=7$ pixels (PCR parameter: RN $=7$ ). (B) Increasing the kernel size to 41 pixels (RN $=2$ ) provides increased visualization of larger structures.

enhancement (RE). Figure 5 shows the effects of changing kernel size. The image in Fig 5A was produced with a PCR kernel size of 7 pixels $(\mathrm{RN}=7)$, whereas $5 \mathrm{~B}$ was generated with a larger kernel of 41 pixels $(\mathrm{RN}=2)$. The larger kernel results in improved visibility of larger structures such as vessels and catheters (Fig 5B). Figure 6 shows the improved visualization of structures in both the lungs and mediastinum after application of the unsharp-masking and gray-scale adjustments in the right image. Figure $6 \mathrm{~A}$ is the left image of a PCR portable chest examination obtained with our derived grayscale parameters. After unsharp masking weighted with the P-curve (PCR parameter $\mathrm{RT}=P$ ) and application of a linear output transform curve $(\mathrm{GT}=A)$, local contrast is increased while maintaining dynamic range. Specifically, note the improved visibility of both mediastinal structures (mitral valve prosthesis) and structures projected in the lung (left subclavian catheter and chest tubes).

\section{DISCUSSION}

Previous investigations of image processing in digital chest radiography were concerned with presenting the radiologist with a single, processed image that was superior to a conventional radiograph. ${ }^{7-10}$ Image processing algorithms were developed that avoided introduction of artifacts or distracting areas that might be counterproduc- tive to film interpretation. For example, marked edge enhancement in the lungs can be distracting to radiologists. ${ }^{7}$ While edge enhancement may increase the detection of pneumothoraces, it may interfere with the interpretation of interstitial diseases by distorting the normal appearance of the pulmonary parenchyma. Therefore, our prior approach to digital chest radiology has been to increase visualization in the retrocardiac and subdiaphragmatic areas through edge enhancement, but to avoid processing in the lungs.

With the introduction of two images on a single film in the PCR system, a different approach may be used. With the techniques described in this report, a gray-scale output transform can be applied to the PCR digital image to result in a left image that is similar to an ideally exposed conventional radiograph. This will be an image with which the radiologist is familiar. The right image can then be more dramatically enhanced to overcome some of the limitations in the conventional image. It is acceptable for this image to look processed or artificial because it is only being used for limited and quite specific applications.

The selection of the appropriate right image will depend on the particular examination and the features that require enhancement. For portable chest radiographs, the presence of a pneumothorax and the positions of tubes and catheters are important observations that are often 

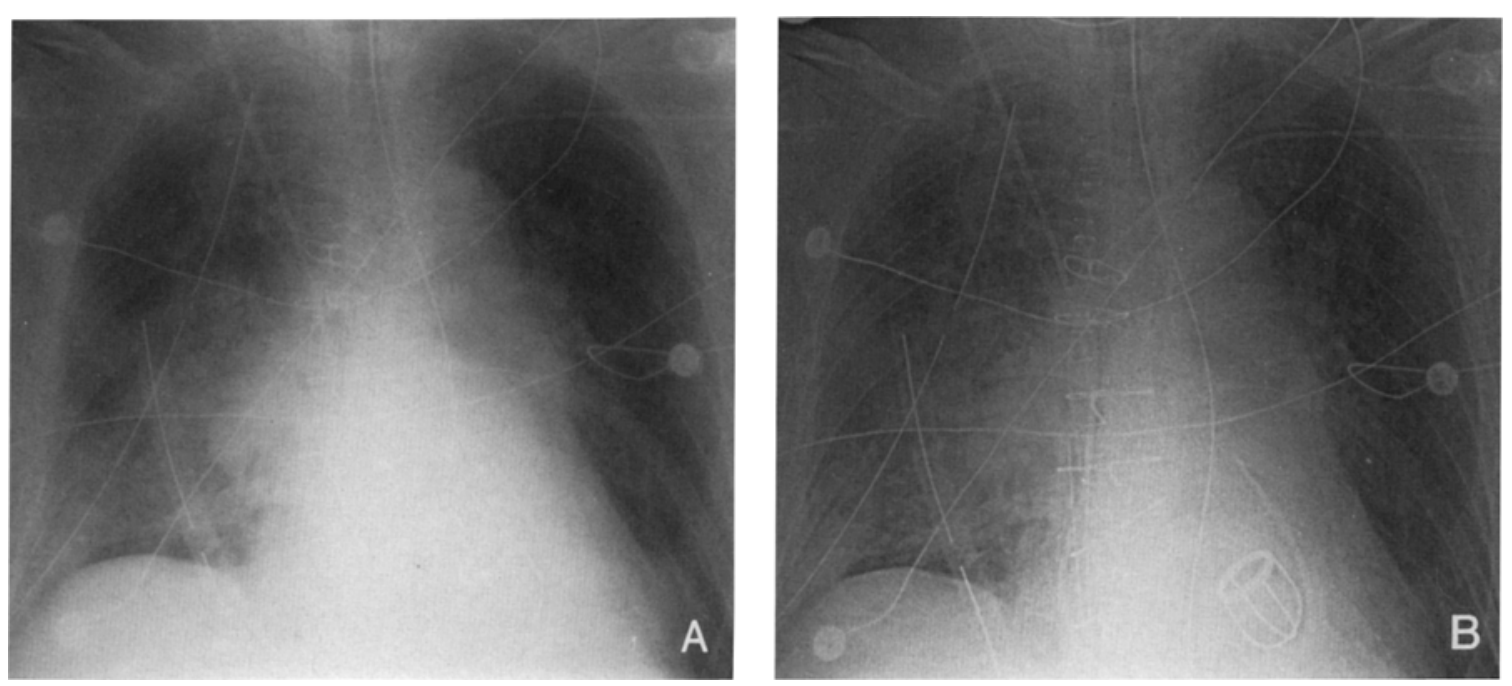

Fig 6. Comparison of the left and right image. (A) The left image of a PCR portable chest examination after implementation of the derived gray-scale parameters. (B) The right image, obtained with unsharp masking using the preferred weighting factor curve (PCR parameter: RT $=P$ ) and a linear gray-scale transform (GT $=A$ ). Note the improved visibility of both mediastinal (mitral valve prosthesis) as well as lung (left subclavian catheter, chest tubes) structures.

difficult to appreciate. ${ }^{11,12}$ Edge enhancement has been shown to increase the detection of pneumothoraces in digitized chest images ${ }^{13}$ and to improve the detection of pulmonary nodules in the retrocardiac and subdiaphragmatic areas. ${ }^{10}$ The right image should then be processed with an edge-enhancing algorithm such as unsharp masking, and the enhancement should be applied in both the lungs and mediastinal regions of the image. The selection of kernel size (frequencies enhanced) and weighting factor (anatomical regions enhanced) for the unsharp-masking algorithm have been discussed by Leh-Nien et $\mathrm{al}^{14}$ and applied to digitized chest radiographs. ${ }^{8,9}$ In general, a larger kernel size and large weighting factor will increase the detection of small square objects superimposed on a background of radiographic noise. The use of small kernel sizes (1 $\mathrm{mm}$ ) and large weighting degrees may actually degrade detectability by enhancing unwanted high-frequency information. ${ }^{14}$ We selected a kernel size (PCR parameter $\mathrm{RN}=3$ ) that is larger than that selected by the manufacturer as the default value $(\mathrm{RN}=4)$.

Computed-radiography/photostimulable-phosphor technology offers exciting new possibilities and has the potential to improve image quality in diagnostic radiology. Because these systems produce digital images, it is important that we understand the options available in printing the images on film or displaying them on monitors. While the systems are installed with preselected parameters for gray-scale adjustment and edge enhancement, these parameters may be changed to meet the needs of the individual radiology department or specific clinical applications. The appropriate selection and documentation of these parameters will also be critical in future observerperformance tests of computed radiography systems.

\section{REFERENCES}

1. Sonoda M, Takano M, Miyahara J, et al: Computed radiography utilizing scanning luminescence. Radiology 148 : 833-838, 1983

2. Merritt CRB, Tutton RH, Bell KA, et al: Clinical application of digital radiography: Computed radiographic imaging. Radiographics 5:397-414, 1985

3. Fuhrman CR, Gur D, Good B, et al: Storage phosphor Radiographs vs conventional films: Interpreters' perceptions of diagnostic quality. Am J Roentgenol 150:1011-1014, 1988

4. Hillen W, Schiebel U, Zaengel T: Imaging performance of a digital storage phosphor system. Med Phys 15:744-751, 1987

5. Sakuma H, Takeda K, Hirano T, et al: Plain chest radiograph with computed radiography: Improved sensitivity for the detection of coronary artery calcification. Am J Roentgenol 151:27-30, 1988

6. Newell JD, Seeley G, Hagam RM, et al: Computed radiographic evaluation of simulated pulmonary nodules: Preliminary results. Invest Radiol 23:267-270, 1988

7. Johnson GA, Danieley N, Ravin CE: Processing alterna- 
tives for digital chest imaging. Radiol Clin North Am 23:335-340, 1985

8. McAdams HP, Johnson GA, Suddarth SA, et al: Histogram-directed processing of digital chest images. Invest Radiol 21:253-259, 1986

9. McAdams HP, Johnson GA, Suddarth SA, et al: Implementation of adaptive filtration to chest radiography. Opt Eng 26:669-674, 1987

10. Sherrier RH, Chiles C, Wilkinson WE, et al: Effects of image processing on nodule detection in digitized chest radiographs: An ROC study of observer performance. Radiology $166: 447-450,1988$
11. Sherrier RH, McAdams HP: Processing portable chest radiographs. Diagn Imaging 8:1 17-118, 1986

12. Greenbaum DM, Marschall KE: The value of routine daily chest $x$-rays in intubated patients in the medical intensive care unit. Crit Care Med 10:29-30, 1982

13. Goodman LR, Foley WD, Wilson CR, et al: Pneumothorax and other lung diseases: Effect of altered resolution and edge-enhancement on diagnosis with digitized radiographs. Radiology 167:83-88, 1988

14. Leh-Nien D, Doi K, Metz CE: Investigation of basic imaging properties in digital radiography. 4. Effect of unsharp masking on the detectability of simple patterns. Med Phys 12:209-214, 1985 